\title{
Active Thermography with Electromagnetic Excitation: Defect-Specific Warming and Underlying Current Flow
}

\author{
by J. Vrana*, M. Goldammer**

\begin{abstract}
*VRANA GmbH, Rosenstraße 6, 83253, Rimsting, Germany, johannes@vrana.net
\end{abstract} \\ **Siemens AG, Otto-Hahn-Ring 6, 81379, Munich, Germany
}

\begin{abstract}
Active thermography with electromagnetic excitation, meaning induction and conduction thermography, is a reliable NDE method with a wide range of applications. Over the past years the basics of the electromagnetic excitation, including the influence of the material of the component to be tested and the necessary post-processing algorithms have been studied.

In this paper a a study on defect models, including delaminations, the well-known slot and notch type cracks, contact-point and "area of reduced conductivity" type cracks, as well as sub-surface cracks is presented. A detailed parameter study is discussed with parameters like depth, width, length, inductor position, rotation, and inclination.
\end{abstract}

\section{Introduction}

A component can be excited in a thermographic inspection in one of two electromagnetic ways, either inductively or conductively $[1,2,3,4,5,6,7]$. Inductive excitation is achieved by an alternating current (AC) running through an inductor placed next to the sample, and conductive excitation is achieved by direct galvanic contacts. In this case, an excitation by means of an alternating (AC) or direct current (DC) is possible. In both methods, a current is created in the electrically conducting material and depending on the current density, heating occurs in the sample. If a crack is present, the current is disrupted, resulting in an alternated current density distribution around the cracked area. Therefore, when heat diffuses to the surface, the crack can be detected by an infrared camera.

During inductive excitation, the current density is highest directly under the inductor (distribution at the surface is described by the proximity effect and the distribution in the depth of the material by the skin effect) and the current flows back in the edges of the front and back surface [1,5]. With respect to DC conductive excitation, if no defects are present, the current density is homogeneously distributed. As for AC conductive excitation, the current density is highest at the edges of the sample (four in the case of a rectangular sample) and decreases in between. This is called the edge effect. Additionally, the skin effect occurs as the current density rises from the middle to the sides [5].

Defects are inhomogeneities in the test material and they change the propagation of both the current and the warming. The two most frequently occurring defect types are delaminations and cracks. Delaminations, as defects that lie parallel to the surface, mainly disrupt the propagation of heat and not of current; cracks, on the other hand, mainly disturb the propagation of current. Because of these effects, defects can eventually be detected by means of an infrared camera.

\section{Delaminations}

The detection of delaminations or, more generally, two-dimensional defects within the test material lying parallel to the surface, take place through various mechanisms, depending on their depth [5].

If the delamination lies (clearly) deeper than the skin depth, an excitation on the surface can be assumed - such as excitation by means of a flash. The heat accumulates between the delamination and the surface, and the surface at this point stays warmer than the surrounding material [8]. However, since the current density distribution on the surface (either induced or through galvanic contact) is generally non-homogeneous, and the thermal flux can also flow to the side, the two-dimensional optical excitation, in which the heat can only diffuse down into the depth, is by far the better excitation option [5].

If the delamination lies within the skin depth (as is almost always the case with CFRP), a two-dimensional heat source on the surface can no longer be assumed but rather a homogeneous warming of the material. In this case, no heat builds up because of a delamination, but the heat is prevented from diffusing to the surface by being held under the delamination, and the surface remains evenly warm or becomes only marginally cooler in the presence of such delaminations. Because of this, and due to the rapid warming of the individual fibers that dominate the image, delaminations are very difficult to detect in this case $[5,9]$.

A third type of delamination occurs between a metal test component and a non-conducting top skin. In this case, the delamination obstructs the diffusion of the heat to the surface that arises in the metal test component. This means that the heat takes longer to diffuse to the surface. Figure 1 shows an experiment in which a detection of this type of a delamination is demonstrated. The phase image of a pulse-phase analysis contains the undamaged part of the test object (red), the delamination (violet) and the place where the non-conducting top skin has separated (yellow). This result from the time the heat needs to diffuse to the surface. However, in this case, too, thermal imaging by flash is the better detection 
method; with it, two-dimensional excitation is possible and there is no inductor to block the field of vision of the infrared camera [5].

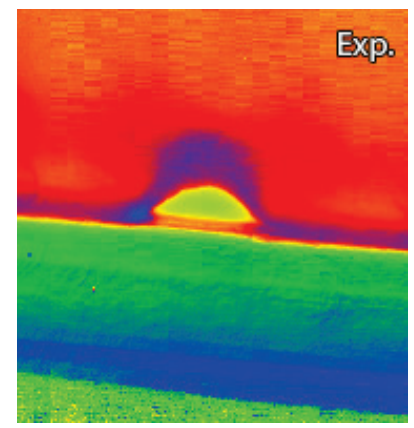

Fig. 1: Delamination of a non-conducting top skin (violet) above an area of flaking (yellow); phase image of the pulse-phase analysis [5].

\section{Simple Crack Models}

Cracks, on the other hand, can be detected well by using thermal imaging with electromagnetic excitation. The two models for cracks mainly found in the literature are a) notches (the model for very long cracks that do not pass through the material) and b) slots (the model for somewhat shorter cracks that do pass through the material). Both crack models and slots with a finite depth (a model for cracks with a certain length and depth profile) are discussed in detail in this chapter.

\subsection{Notch Type Cracks}

Long surface cracks, such as occur in the hot-rolling of steel bars or during the drawing of steel wires (mainly in the longitudinal direction) or crack where the current flows beneath the crack due to the nature of the inhomogeneity are a defect types that can most easily be modelled by a notch (ref. to Figure 2a).

For the induction thermography testing of this type of cracks, an inductor is set up at right angles to the normally elongated longer test component. For an evaluation of the whole test component, the specimen can be moved underneath the inductor and the camera; this makes it possible to examine the entire test component for notches in the longitudinal direction [3].

The change in current-density distribution caused by the notch is shown in Figure $2 \mathrm{~b}$. The current density is clearly decreased at the edges between the upper surface and the shoulders of the notch (the extent of this zone depends on the skin depth) and increased at the two edges between the shoulders and the floor of the notch. This is caused as the current must flow "underneath" the notch.

Due to the current density distribution the inside of the crack is warmer and the edges colder than the surrounding material during the excitation (most notch-like real cracks do not provide a view into the crack, thus, real cracks will only show colder edges). However, the heat from the bottom, as it diffuses to the surface, is restricted into one dimension by the crack, and, the conclusion of the induction pulse the top edges tend to stay warmer than the surrounding material $[4,5]$.

In the case of a low skin depth, the current density flows in a narrower zone, so the current-density distribution at the corner is narrower and therefore higher. In this case, the corner heats up more quickly than in the case of thicker skins. If the skin is very thin (e.g.: in the case of ferromagnetic materials), it appears that the corner becomes instantaneously warmer than the surrounding material [5].

\subsubsection{Dependence of the Current Density and Temperature Distribution from the Notch Depth}

Figure $2 c$ shows the current density along the notch shoulder, for notches of different depths. The current density on the upper surface (at $x=0$ ) decreases with greater notch depths and no longer changes when the notch depth is one skin depth or more. Starting with a notch depth of around 2 skin depths, a zone along the notch shoulder can be seen in which the current density is relatively constant. For deep notches (from around 2 skin depths), the current-density distribution can therefore be divided into three sections:

On the surface of the test component, there is a zone of reduced current density that reaches to a depth of about one skin depth. This is the zone in which the current is deflected through $90^{\circ}$ by the notch. Underneath, the current flows in the case of notches deeper than about 2 skin depths, along the x-axis; this leads to a relatively homogeneous currentdensity distribution in this zone of the notch shoulder. On the inner edge, the current can again flow in the direction predetermined by the excitation (z-axis) and this results in excessive current density [5].

This behavior can be modelled by area sources along the notch shoulders (between the depth of one skin depth and the depth of the notch) and line sources at the inner edges [5]. 

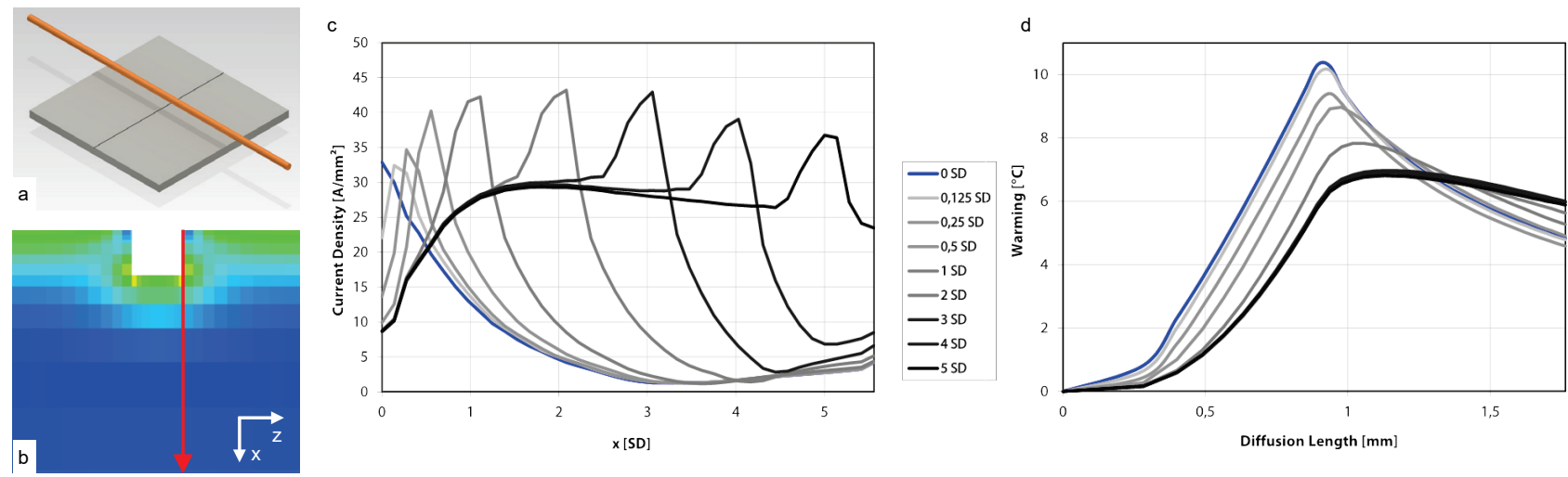

Fig. 2: a\&b) Model for a notch like crack

b) Distribution of the current density in depth (from simulation calculations for notches 0 to 5 skin depths deep and a skin depth of 0,9 mm; test component thickness: $5 \mathrm{~mm}$ ) along the line drawn in the sketch (c)

b) Development of the warming on the surface directly next to the notch for notches of different depths; the time was adapted to the appropriate diffusion length (the figure shows the result of simulation calculations on Inconel with a $100 \mathrm{~ms}$ induction pulse - this corresponds to a diffusion length of around 0,9 mm) [5].

The current-density distributions for notches of different depths presented in Figure $2 \mathrm{c}$ give us the temperature developments on the surface directly next to the notch (shown in Figure 2d). As discussed above the current density on the surface decreases as the notch depth increases, up to about one skin depth. Starting from one skin depth, the currentdensity distribution changes only in the depth (not on the surface). So, up to a notch depth of one skin depth, the effect of the notch depth on warming can be seen immediately after the start of the induction pulse. Deeper notches can only be differentiated by observing the long-term temperature development [5].

\subsubsection{Dependence of the Current Density Distribution from the Notch Width}

Figure 3a shows the current density distribution in the bottom of the notch depending on the notch width. It also shows the current-density distribution on a specimen with a $100 \mathrm{~mm}$-wide notch in red, meaning a $100 \times 100 \mathrm{~mm}^{2}$ test component on which the entire surface has been removed to one notch depth and represents the distribution induced by the inductor at this enlarged distance.

The current density between the edges of wide notches falls to the level of the profile reduced by one notch depth; in other words, the current-density distribution on the floor of the notch overlaps the distribution induced by both edges of the notch and the distribution induced by the inductor at this distance. The rise in current density induced by the notch edges is greater the narrower the notch. For very narrow notches, this rise is around double that for wide notches. This is a result of the increasing overlapping of the distribution emanating from the right and left notch edges [5].
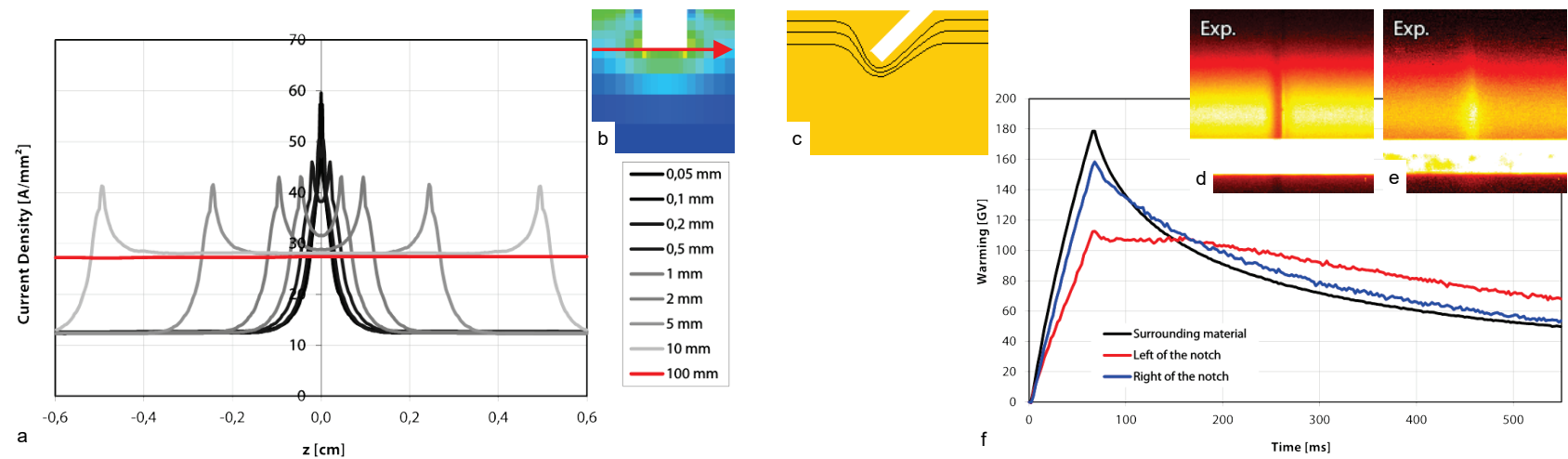

Fig. 3: a) Current-density distribution (calculated by simulation) in the floor of the notch for notches 0,05 to $10 \mathrm{~mm}$ wide, along the line drawn in the sketch (b). The current-density distribution can also be seen (in red) for a specimen profile that has been reduced by one notch depth ("100 $\mathrm{mm}$ wide notch").

c) Plotted current flow lines for a tilted notch; $d$-e) Signal from a notch approx. $1 \mathrm{~mm}$ wide that runs at an angle of $45^{\circ}$ in the material, at an induction pulse of $60 \mathrm{~ms}$. Image of the result after d) $60 \mathrm{~ms}$ and e) $300 \mathrm{~ms}$;

f) Warming on the left (red) and right (blue) adjacent to the notch in comparison to the warming of the surrounding material (black) [5]. 


\subsubsection{Current Density and Temperature Distribution for Tilted Notches}

In the case of notches that are not perpendicular to the surface but at an angle, the current-density distribution changes. The current is better able to follow the notch or to be more exact, as it can be seen in Figure 3c, the current on the right-hand side can follow the notch profile better while the current on the left cannot follow it as well. Therefore, the effect of reducing the current density is weakened on one side with greater angles and strengthened on the other side.

Fig. $3 \mathrm{~d}-\mathrm{f}$ shows the result of an induction thermography investigation of a tilted notch at an angle of $45^{\circ}$ (as usual: obliquely from above, to show the area directly under the inductor). On the image after $60 \mathrm{~ms}$ and at the time series, the effect on the current density on the outer notch edge can clearly be seen [5].

Furthermore, the effect of the slower cooling also changes, since the point of highest current density is displaced to the inner edge of the notch. As it can be seen in Fig. 3d-f, the side with the smaller angle remains warm longer (lefthand side) than the surrounding material, longer than the other side and longer than not-tilted notches.

\subsubsection{Detection Sensitivity Dependence on the Angle between the Applied Current Flow Direction and the Notch}

In all the considerations above, it has been assumed that the angle between the inductor and the notch is $90^{\circ}$, as the current flow is most disturbed at that angle.

Figure 4 shows the result of an inductively excited notch (excitation time: $50 \mathrm{~ms}$ ) rotated by $90^{\circ}, 60^{\circ}, 30^{\circ}$ and $0^{\circ}$ after $50 \mathrm{~ms}(\mathrm{a}-\mathrm{d})$ and $170 \mathrm{~ms}$ (e-f). At an angle of $90^{\circ}$ and after $50 \mathrm{~ms}$, the outer notch edges are cooler than the surrounding material, but the floor of the notch is warmer. After $170 \mathrm{~ms}$, as discussed in 3.1.1, the outer notch edges stay warmer than the surrounding material. As the angle is reduced, the current finds it easier to follow the course of the crack and the current density at the outer notch edges (the zone of reduced current density) increases. So, at smaller angles, the effect that after $50 \mathrm{~ms}$ the zone adjacent to the notch appears cooler becomes less until, at $30^{\circ}$, it is almost negligible. At $0^{\circ}$, a slight warming next to the notch can be seen. This is the result of the edge effect that occurs at both notch edges. However, the typical effect after a longer period of time $(170 \mathrm{~ms})$ can be seen at all angles. At an angle of $0^{\circ}$, this is not a result of the heat that diffuses from the inner notch edges, but of the propagation of the heat that occurs at the outer notch edges due to the edge effect.
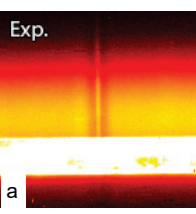

b
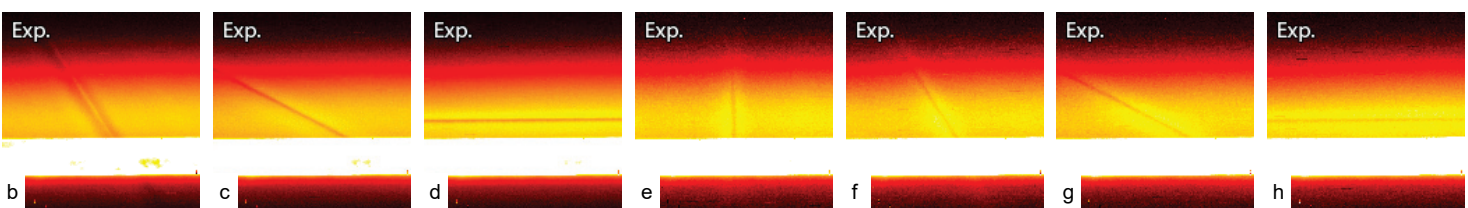

Fig. 4: Thermal image taken with the notch (approx. $1 \mathrm{~mm}$ wide) rotated through $90^{\circ}, 60^{\circ}$, $20^{\circ}$, and $0^{\circ}$ (from left to right) after $50 \mathrm{~ms}(\mathrm{a}-\mathrm{d})$ and $170 \mathrm{~ms}(\mathrm{e}-\mathrm{h})$ at an induction pulse lasting $50 \mathrm{~ms}$. The results of the experiments were recorded by infrared camera mounted to point downwards at an angle so that the zone directly under the inductor was visible. The inductor is the light-coloured bar visible in the images. [5].

\subsection{Slot Type Cracks}

A crack can be described by its specific length and its depth profile. Therefore, depending on the nature of the crack the current flows beneath the crack or around the crack tips. To model this behavior, it is best to look at these two alternatives individually. The case where the current flows beneath the crack was discussed in the last section by using a model geometry infinite in length but finite in depth: a notch. The case where the current flows around the crack tips is given by a slot which is finite in length but extends completely through the sample.

Figure $4 a-b$ shows a model of slot type cracks. Figure $4 a(0,1 \mathrm{~mm}$ wide) and $4 \mathrm{c}(2 \mathrm{~mm}$ wide) show the currentdensity distribution on the surface of the test component and $4 \mathrm{~b}$ in the depths. In the zone around the slot a local currentdensity disturbance is visible: in the environment of the slot a clear excess at the tip of the crack (B) and a reduction in the slot shoulders (A) are present. Both effects are, as in the case of the notch, a result of the current excited from outside in the z-direction which is blocked by the slot and must therefore flow round it. This causes a reduction in the current density in the middle of the slot shoulder (like at the outer notch edges), since the current is deflected. At the same time, the current density increases at the tips of the slot (like at the inner edges of the notch), since in this case the entire current flows round the slot. In contrast to the notch, the zones in which the current density is mostly changed are not found inside the specimen but on the surface and the warming does not have to diffuse to the surface. There is therefore no temporal interrelation up to the increase in blur generated by diffusion.

\subsubsection{Dependence of the Current Density Distribution on the Slot Width}

The dependence of the current density distribution on the slot width goes parallel to the dependence on the notch width discussed in 3.1.2. In Figure 4a vs. 4c, the change of the current-density distribution at a slot with a width of $0,1 \mathrm{~mm}$ 
(Fig. 4a) is compared with the one of a $2 \mathrm{~mm}$ wide slot (Fig. 4c). The tips of the $2 \mathrm{~mm}$ wide slot (B) are also heated; but, since the slot is wider, there is (like in the example of the notch) a zone of reduced current density (C) between the two edges of a slot tip. This phenomenon can be explained by analogy with 3.1.2: the composition of the current density in the area of the slot tips is the addition of the undisturbed current density and the current-density distributions generated by both edges of the slot. (Notch: current density in the bottom of the notch plus the current-density distributions generated by both edges of the notch) [5].
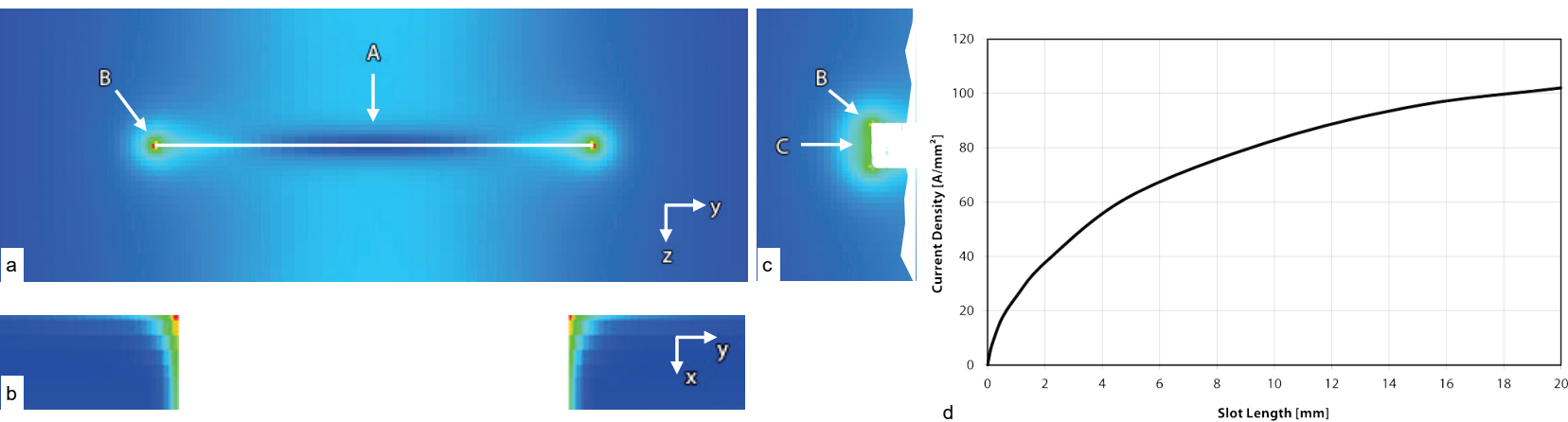

Fig. 4: a) z-y view; b) $x-y$ view: Change in current-density distribution caused by a slot 0,1 mm wide by $20 \mathrm{~mm}$ long and with thickness a the component of $5 \mathrm{~mm}$ (simulation calculation with an inductor placed along the z-axis)

c) section of the z-y view for a slot $2 \mathrm{~mm}$ wide and $20 \mathrm{~mm}$ long

d) Increase in current density at the tip of the slot (B) for slots of different lengths (simulation) [5]

\subsubsection{Dependence of the Current Density Distribution on the Slot Length}

As Figure 4b shows, the distribution of the current density towards the bottom of a slot does not correspond to the normal exponential decrease due to the skin effect, but the current is distributed over a larger area in depth (in the vicinity of the slot). This area increases with greater slot length, based on the exponential decrease that is prevalent in the undisturbed case.

This means that the growth of the current density at the slot tips on the slot length cannot simply be calculated by integration through the distribution of current density given by the proximity effect. Rather, the current density grows slower. Several simulation calculations were carried out to compute the increase in current density at the tips of the slot, depending on the slot length. Figure 4d shows the result of these calculations. In order to apply the effect of raising the current density through the slot, the current density of a test component without a slot in the same place was deducted [5].

\subsubsection{Dependence of the Detection Sensitivity on the Angle between Specified Current-Flow Direction and Slot}
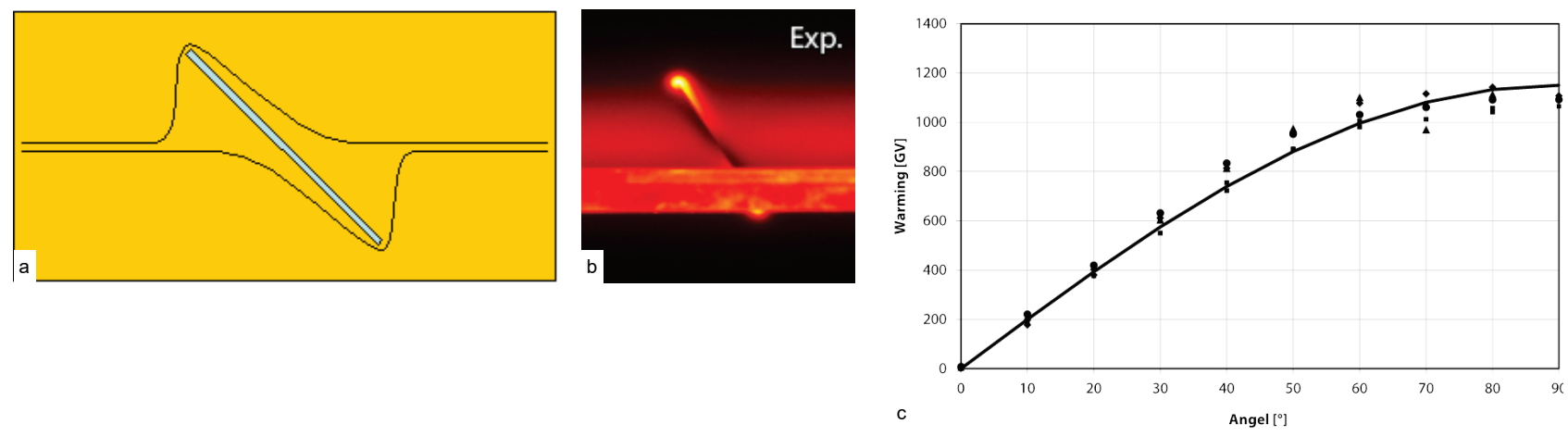

Fig. 5: a) Sketch of current flow lines of a rotated slot b) Thermal image of a slot rotated by $60^{\circ}$ c) Temperature rise at the slot tips depending on the rotation obtained by experiment [5].

Rotated slots show, like tilted notches, a flow against effect (Figure 5a). This leads to the result in Fig $5 \mathrm{~b}$ which represents the induction thermography image of a slot rotated by $60^{\circ}$. Here warming on one side of the slot and cooling on the other is visible $[4,5]$.

Fig. $5 \mathrm{c}$ shows the temperature at the slot tip depending on the angle minus the temperature obtained on an undamaged test component at this same point. As expected the correlation between the angle and warming is sinusoidal. 
For arbitrarily oriented cracks (notch-type cracks as well as slot-type cracks) two investigations must be performed with both inductive and conductive (galvanic) coupling with various current-flow directions (preferably offset by $90^{\circ}$ ).

\subsubsection{Dependence of the Detection Sensitivity on the Position of the Inductor}

In the case of induction thermal imaging investigations, the highest current densities are generated directly under the inductor. Therefore, the dependence of the position of the inductor is of interest in terms of detection sensitivity.

Figure 6 shows three experimental results of images taken by infrared camera (obliquely from above, to show the area directly under the inductor). In Fig 6a, the center point of the $20 \mathrm{~mm}$-long slot is immediately below the inductor; in Fig. $6 \mathrm{~b}$, the center point has been moved $10 \mathrm{~mm}$, so one of the slot tips is directly below the inductor; and in Fig $6 \mathrm{c}$, this point has been displaced by $20 \mathrm{~mm}$. At $0 \mathrm{~mm}$, both slot tips can be seen clearly; at $10 \mathrm{~mm}$, one tip can be seen very well and the other almost not at all; and finally, at $20 \mathrm{~mm}$, only the lower one is visible. For improved detectability of all the slots the phase image of a pulse-phase analysis can be used (see Fig. 6d-f) [5].

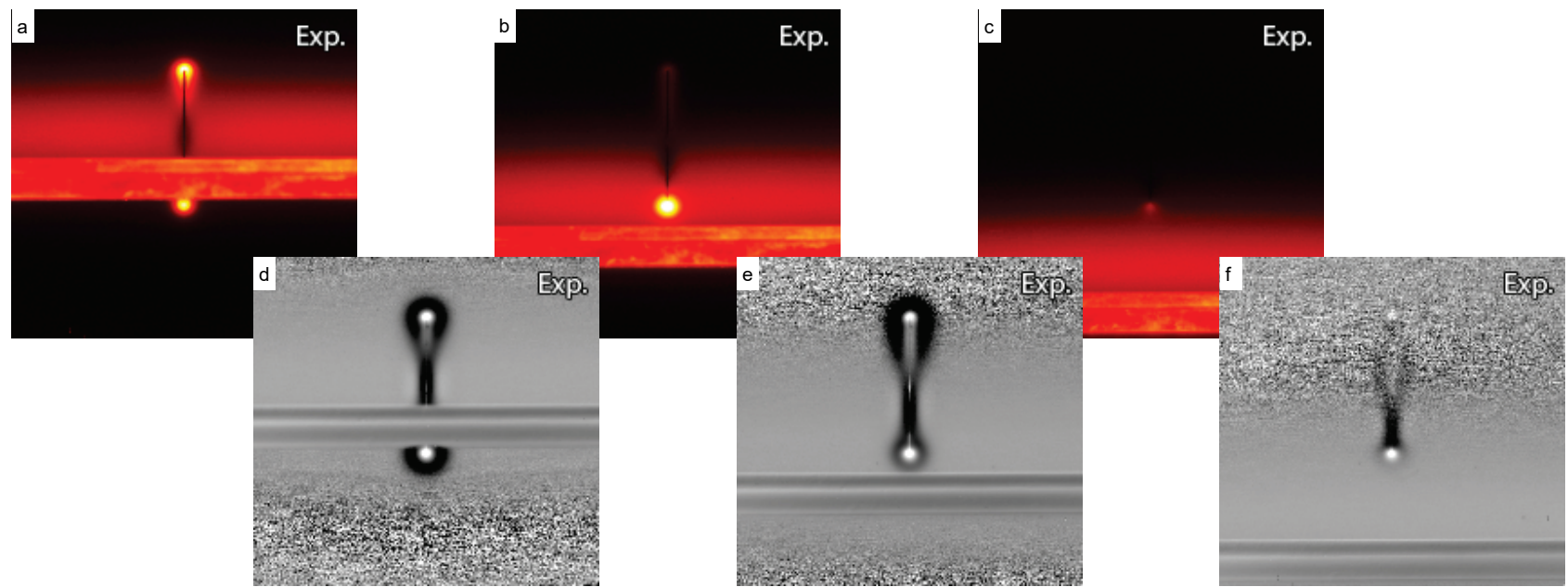

Fig. 6: A 20-mm slot a) directly under the inductor; displaced by b) $10 \mathrm{~mm}$ and c) $20 \mathrm{~mm}$. $d$-f) Results (phase image) of the pulse-phase analysis [5].

\subsection{Detection of slots with Finite Depth}

After discussing the effects of notch and slot like cracks independently (in section 3.1 and 3.2) slots with a finite depth profile are considered which show, depending on the length and depth profile of the crack, signatures from both basic cracks models.
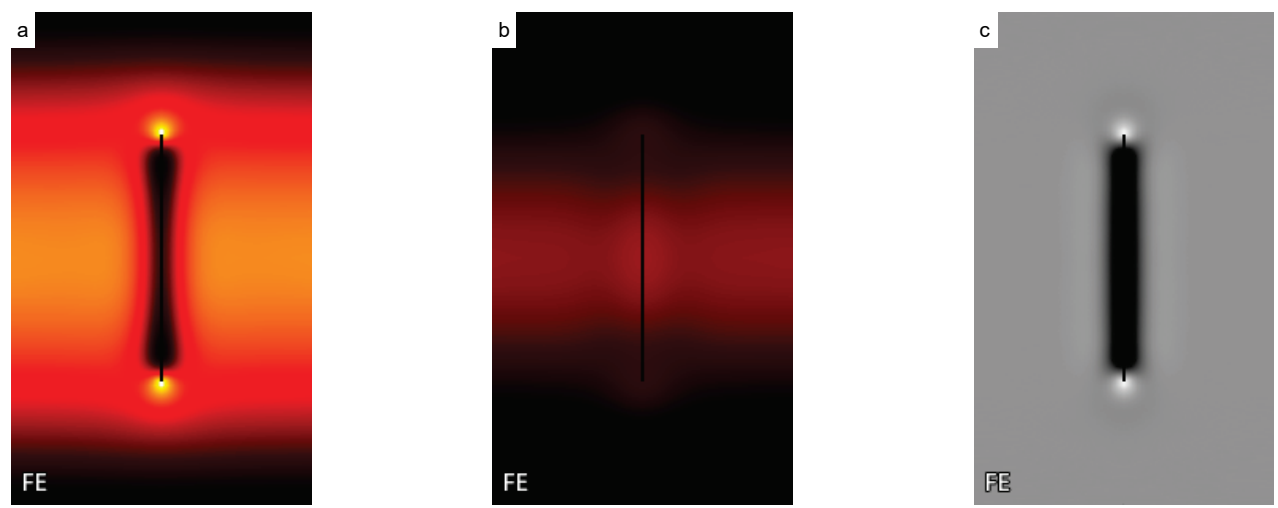

Fig. 7: Simulated result of an examination by induction thermography of a slot $10 \mathrm{~mm}$ long, 0,1 mm wide and 0,9 mm deep (corresponding to one skin depth) with an induction pulse lasting for $50 \mathrm{~ms}$;

a) after $50 \mathrm{~ms}, b)$ after $200 \mathrm{~ms}, \mathrm{c})$ phase image of the pulse-phase analysis [5].

Figure 7 shows the result of an investigation on a test component containing a slot with a depth of one skin depth. This test component was heated by inductive excitation for $50 \mathrm{~ms}$. The image after $50 \mathrm{~ms}$ shows that the slot tips are 
warmer and the slot shoulders cooler than the surrounding material; in other words, the typical signal from a slot. However, compared with Fig. 6a, the area of lower temperature along the slot shoulders is longer and the temperature at the slot tips lower. Both result from the effect that the current, because of the finite depth of the slot, can also flow under the slot. The image after 200 ms shows that, after the end of the induction pulse, the slot shoulders cool down less than the surrounding material. This is the result of the fact that the heat coming from the inner slot shoulders is diffused to the surface, as in the case of notches. The phase image of the pulse-phase analysis clearly demonstrates this delayed effect typical for notchlike cracks.

\section{Enhanced Crack Models}

Notches and slots give good fundamental models for a basic understanding for defect detection using thermographic methods with electromagnetic excitation. Previous publications $[1,4,5]$ have shown that for an extended understanding of defect signals enhanced crack models like the contact point or the conductivity model are necessary.

\subsection{Contact Point Mode}

Slot type cracks, discussed in 3.2, show heat spots only at the end of the slot. Real cracks, depending on the morphology, can give a result showing several heat spots. Those can be explained by contact points $[1,4,5]$. This signal causing mechanism is also the reason why both cracks with high and low residual stresses can be detected with induction and conduction thermography in contrast to many other NDE methods [4].

\subsubsection{Dependence of the Detection Sensitivity on the Angle between Specified Current-Flow Direction and Contact Point Type Cracks}

A reduced angle between the inductor and contact point type cracks (see Figure 8) leads to a lower amplitude of the indication (as in the case of notches and slots). At $0^{\circ}$, the spaces between the holes remain cooler than the undisturbed material and the area above and below the holes warms up more strongly. This is only an effect of the hole diameter, since the current must flow past the holes. This means that the current density rises next to the holes and, because of the small gap between the holes, the current does not flow back into the area between the holes.
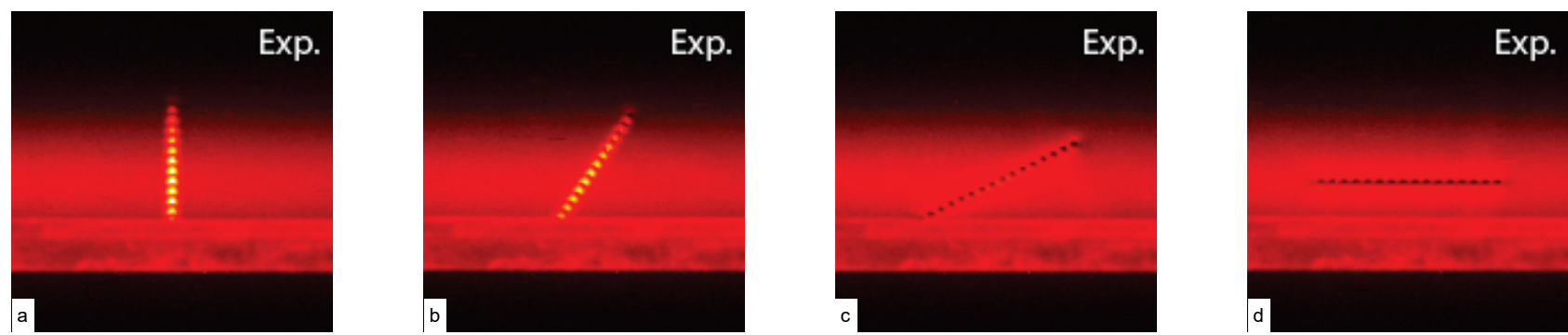

Fig. 8: Dependence of the contact point model indication (16 $1 \mathrm{~mm}$ diameter holes drilled in a line close to each other) on the rotation a) $90^{\circ}$, b) $60^{\circ}$, c) $30^{\circ}$, and d) $0^{\circ}$ (in these images, the inductor can be seen as a red bar) [5].

\subsection{Conductivity Model}

For cracks with high residual stress, the density of contact points can be higher than what can be resolved by thermography. In such cases, a crack can alternatively be deemed a zone of reduced electric conductivity. In the following the conductivity model is discussed for both notch and slot type cracks.

\subsubsection{Conductivity Model for Notch Type Cracks}

Figure 9 shows the results of a simulation (current density and warming) in which the notches are filled with

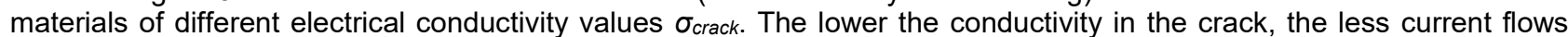
directly at the surface and the more current around the inner notch edges. So, at a slightly weakened conductivity value (Fig. 9c-d) the crack heats up along its entire length (the current density is actually lower in the crack, but the warming is greater due to the increased resistance). For even lower conductivity values (Fig. e-j), the warming of the material in the crack is weaker but, the typical effect of the notch (the two cooler edges adjacent to the notch) becomes clearer and clearer until finally, as in the case of the notch (Fig. 9k-I), this effect is all that is seen [5]. 


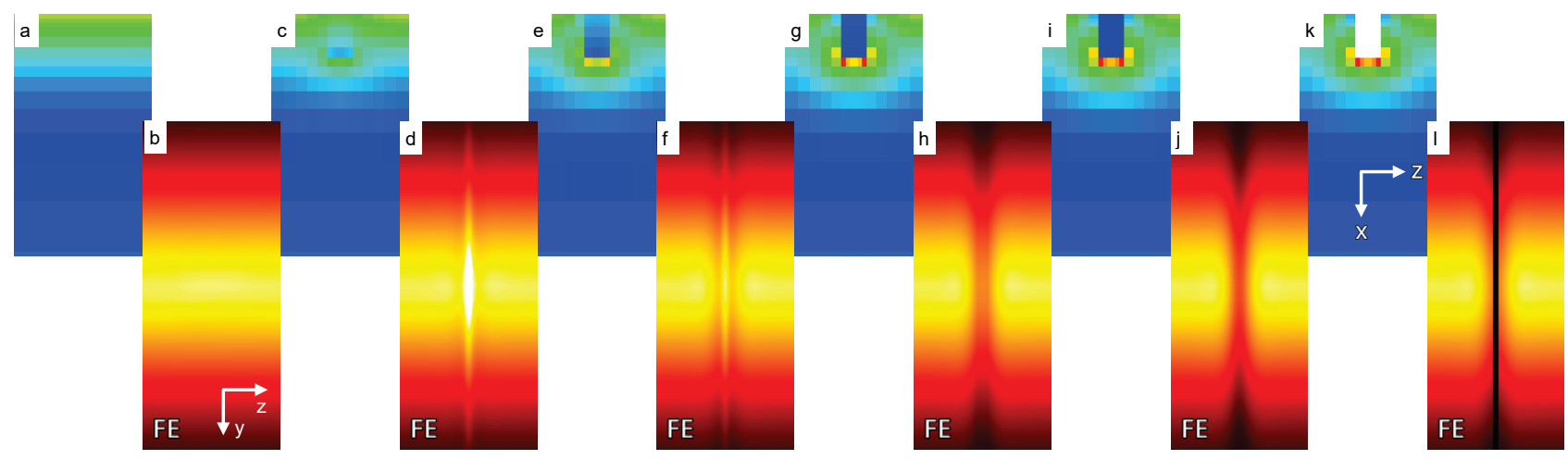

Fig. 9: Current-density distribution inside the crack ( $a, c, e, g, i, k)$ and the applicable images of warming from above (b, $d, f, h, i, l)$ of a $0,5 \mathrm{~mm}$ wide and $0,9 \mathrm{~mm}$ (1 skin depth) deep notch filled with a material whose electrical conductivity is not the same as that of the basic material. The inductor (blanked out here) is set up horizontally and lies across the middle of the thermal image above the test component.

$\left.a-b) \sigma_{\text {crack }}=\sigma_{\text {specimen, }} c-d\right) \sigma_{\text {crack }}=0,25 \sigma_{\text {specimen, }}$ e-f) $\sigma_{\text {crack }}=0,016 \sigma_{\text {specimen, }}$ $g$-h) $\left.\left.\sigma_{\text {crack }}=0,0063 \sigma_{\text {specimen, }} i-j\right) \sigma_{\text {crack }}=3,910^{-3} \sigma_{\text {specimen, }} k-l\right) \sigma_{\text {crack }}=0[5]$.

\subsubsection{Conductivity Model for Slot Type Cracks}

Figure 10 shows the results of a simulation (warming) in which the slots are filled with materials of different

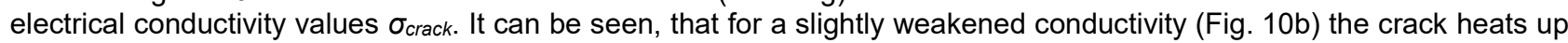
over its entire length (as in the case of slot type cracks discussed in 4.2.1). If the conductivity is significantly weakened (Fig. 10c), the crack heats up more strongly for its entire length, but since part of the current flows around the crack tips, these also heat up. If the electrical conductivity is very much weakened (Fig. 10d), the only warming occurs at the crack tips, as in the case of a slot (Fig. 10e), and the area in between remains cold. Figure 10f shows the warming in the middle of the crack and at the crack tips, as a function of the relative electrical conductivity of the model crack $[1,4,5]$.
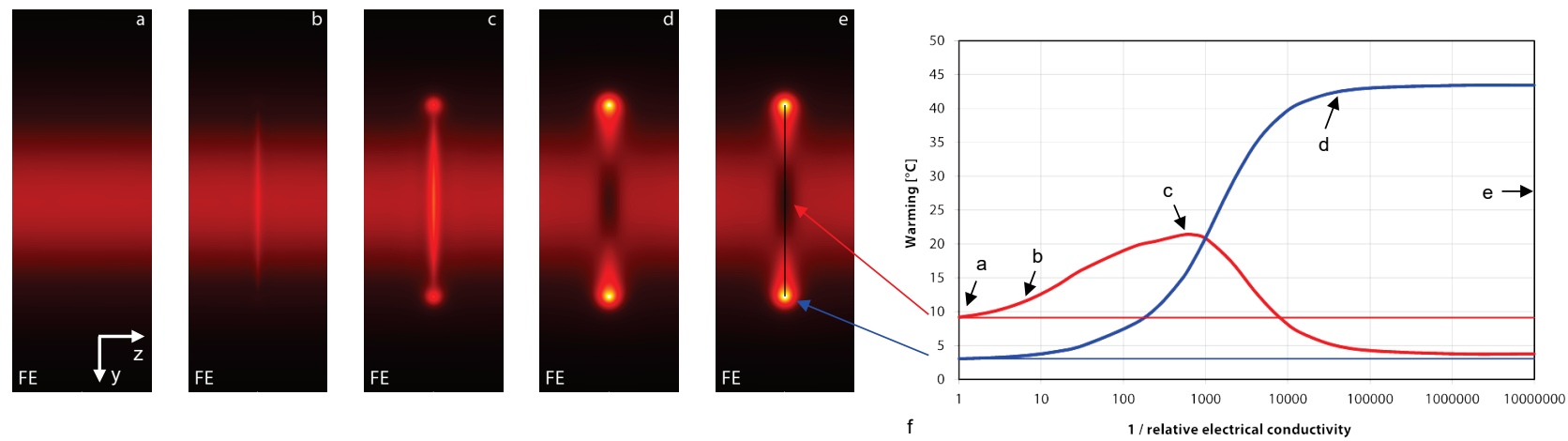

Fig. 10: Calculated simulated images of an induction thermographic investigation of a $20 \mathrm{~mm}$ long by $0,1 \mathrm{~mm}$ wide slot filled with a material whose electrical conductivity is not the same as that of the basic material. The inductor

(blanked out here) is set up horizontally and lies across the middle of the image above the test component.

a) $\sigma_{\text {crack }}=\sigma_{\text {specimen, }}$ b) $\sigma_{\text {crack }}=0,13 \sigma_{\text {specimen, }}$ c) $\sigma_{\text {crack }}=1,410^{-3} \sigma_{\text {specimen, }}$ d) $\sigma_{\text {crack }}=1,510^{-5} \sigma_{\text {specimen, }}$ e) $\sigma_{\text {crack }}=0$

f) Temperature rise at the crack tips (blue) and in the middle of the crack (red) as a function of the rise in the electrical resistance of the crack in comparison with the warming of the test component without crack (thin lines). [1,5].

\subsection{Sub-surface Cracks}

Simple and enhanced models discussed so far assume cracks that reach to the surface of the test component. Hidden cracks in the material cause the same warming effects as cracks open to the surface (heating at the crack tips, contact points or areas of lower electrical conductivity). However, since this warming takes place inside the material, it is weaker because of the lower current density (skin effect) and due to the necessity, that the heat needs to diffuse out to the surface.

In addition to those conventional warming effects, hidden cracks that are not too deep in the material are subject to another direct heating effect. As in all defects, the current must flow around the crack. On the one hand, this generates 
the warming effects already mentioned and, on the other, the current can also travel above the crack, which increases the heating energy in this region. This warms the material above the crack, and the entire length of the crack can be seen in the infrared image (ref. to Figure 11a). Fig. 11b-c show experimental results on circular slots cut from the backside with a residual thickness of material equal to quarter (1), half (2), one (3), one and a half (4), and two skin depths (5). In the images representing the maximum signal intensity (Figure 11b) only the first two are visible (and the third very weekly). Using the phase image of a pulse-phase analysis also slot \#3 and \#4 become detectable. However, as the black color shows the signal is delayed. This is caused by a different detection mechanism: the heat is accumulated between the crack and the surface. This means that, after the induction pulse, the point where the crack is located cools down more slowly. The extent of this effect does not depend on the skin depth, but on the physical length of thermal diffusion; this, in turn, depends on the surface area that the slot or crack has to offer against the distribution of the heat. So, closed cracks (in contrast to open cracks as investigated here) deeper than 0,5 to 1 skin depth will only cause a minimal accumulation of the heat and will most likely not be detectable [5]. Summarizing: both simulations [5] and experiments (Figure 11b-c) have shown that sub-surface cracks up to approximately 0,5 to 1 skin depth are detectable and cause a different image compared to indications open to the surface.
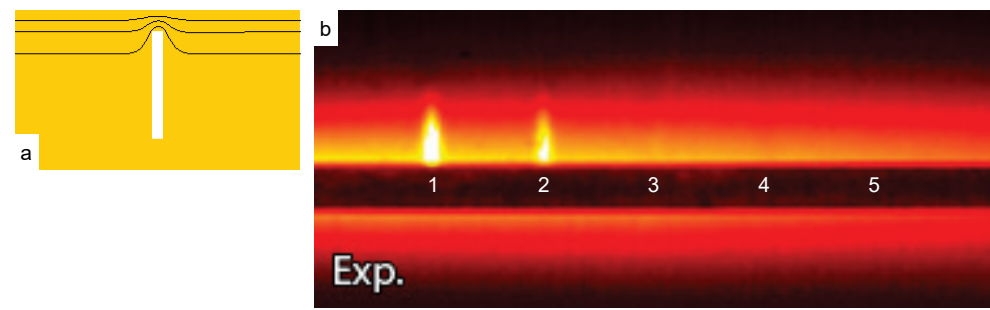

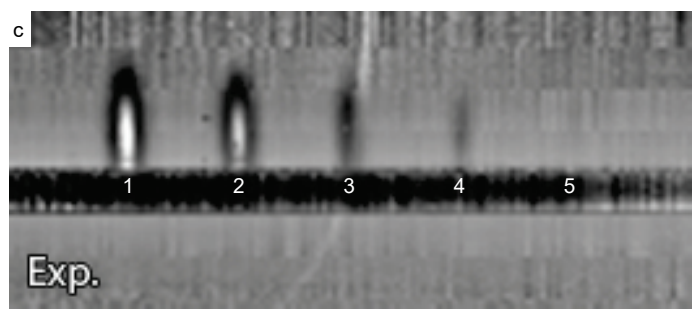

Fig. 11: a) Sketched current flow lines on a sub-surface crack b-c) Experimental detection of sub-surface slots (approx. 0,5 mm wide) with a residual wall thickness of 1) 0,25 skin depths 2) 0,5 skin depths 3) 1 skin depth 4) 1,5 skin depths and 5) 2 skin depths (from left to right); b) moment of maximum signal intensity; c) phase image of a pulse-phase analysis; the inductor can be seen in the images as a black bar [5].

\subsection{Hidden Cracks under Non-Conductive Coatings}

Cracks hidden by a non-conducting coating lead to no additional heating effect. Warming takes place as usual through the effects discussed above: inner notch edges, crack tips, contact points, areas of lower conductivity, and, if they are also covered by a conductive coating, through warming above the crack.

This warming cannot be detected directly but must first diffuse to the surface. Depending on the thickness of the coating and the diffusivity of the material, the signal becomes more diffuse and weaker. To investigate this phenomenon more closely, simulations using different coating thicknesses were conducted (zirconium oxide); the induction pulse was $100 \mathrm{~ms}$ in each case.
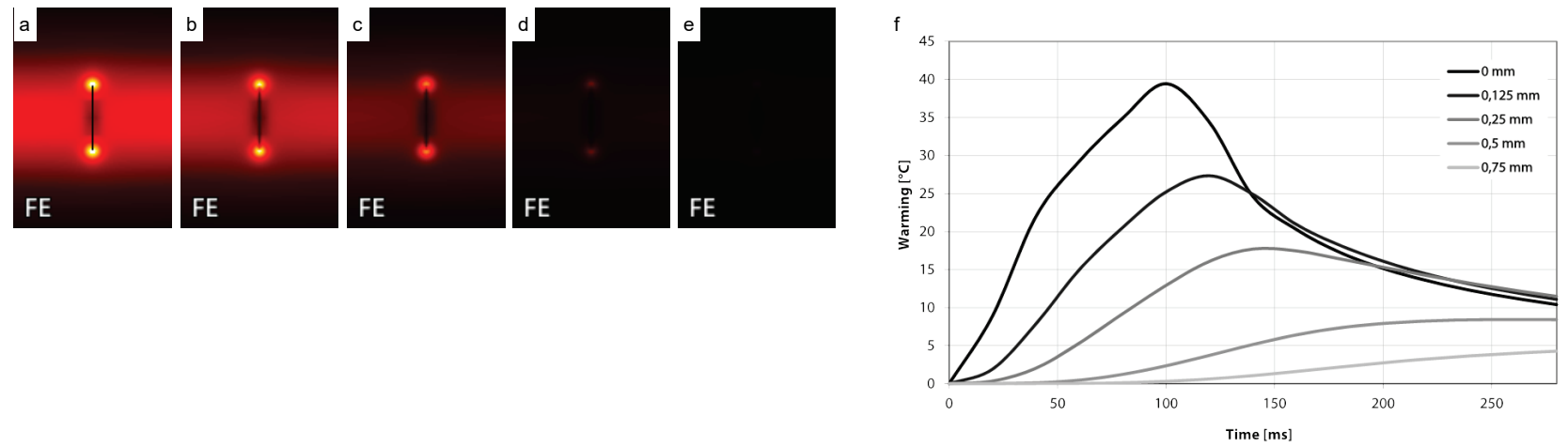

Fig. 12: Simulated warming of a slot $\left(10 \times 0,5 \mathrm{~mm}^{2}\right)$ under a coating (100 ms induction pulse);

a) without coating; b) 0,125 $\mathrm{mm}$; c) 0,25 $\mathrm{mm}$; d) 0,5 $\mathrm{mm}$; e) 0,75 $\mathrm{mm}$ tick zirconium oxide coating.

The blanked out inductor is set up horizontally and lies across the middle of the image above the test component. f) Development over time of the warming at the slot tips for the various coating thicknesses (simulation) [5].

Figure 12a-e shows the infrared image directly after the end of the induction pulse; the dependence on the coating thickness, described above, can be clearly discerned. Fig. $12 \mathrm{f}$ goes on to show the time series of the warming at the slot tips where, in addition to the effect that the warming on the surface becomes less and less, it can be seen that the heat 
also takes longer to diffuse from inside out to the surface. This leads to the following correlation between the coating thickness and the time $t$ after the induction pulse up to maximum warming on the surface (diffusivity $\alpha$ ):

$$
d \approx 1.6 \sqrt{\alpha t}
$$

The length of time during which the infrared camera is still recording after the induction pulse should be adapted to the maximum coating thickness; or, alternatively, the delay in the thermal signal can be used to determine the coating thickness. The pulse-phase analysis can be used to improve the signal-to-noise ratio (even where the duration of recording is not matched to the coating thickness) or to determine the coating thickness.

\section{Summary and Outlook}

Active thermography using electromagnetic excitation can be applied inductively and conductively and is ideally suited for the detection of cracks close to or open at a surface making for easy inspection. Using mechanics such as robotics, translation or rotary stages, automated inspection can be implemented for serial production. The thermographic technique has the advantage that it is contact-free, without coupling media, and without chemicals. UV (ultraviolet) illumination is not necessary. Image generation is part of the detection process. This allows further post-processing which allows for automated indication detection.

The models for crack detection and the parameter studies presented allow for a clear understanding of the thermographic analysis of real cracks. They also confirm that induction and conductions thermography are ideally suited for all kinds of surface or close to surface cracks, including cracks with low or high residual stress which are otherwise difficult to detect via acoustic thermography or penetrant testing. The fact that this process can be automated shows the value of thermography with electromagnetic excitation.

\section{REFERENCES}

[1] Vrana J., Goldammer M., Baumann J., Rothenfusser M., Arnold W., "Mechanisms and Models for Crack Detection with Induction Thermography". AIP Conference Proceedings, vol. 975, pp. 475 - 482, 2008, DOI: 10.1063/1.12902698.

[2] Vrana J., Goldammer M., Bailey K., Rothenfusser M., Arnold W., "Induction and Conduction Thermography: Optimizing the Electromagnetic Excitation Towards Application”. AIP Conference Proceedings, vol. 1096, pp. 518 - 525, 2009, DOI: 10.1063/1.3114299.

[3] Goldammer M., Mooshofer H., Rothenfusser M., Bass J., Vrana J., "Automated Induction Thermography of Generator Components". AIP Conference Proceedings, vol. 1211, pp. 451 - 457, 2010, DOI: 10.1063/1.3362428

[4] Vrana J., Goldammer M., "Induction and conduction thermography: From the basics to automated testing taking into account low and high residual stresses". Materials Testing, to be published, 2018.

[5] Vrana J., "Grundlagen und Anwendungen der aktiven Thermographie mit elektromagnetischer Anregung Induktions- und Konduktionsthermographie". Shaker, Herzogenrath, Germany, 2009, DOI: 10.13140/RG.2.1.1680.4567.

[6] Vrana J., Goldammer M., Netzelmann U., "Induction and Conduction Thermography: A new Surface Inspection Method Suited for the Forging Industry". 20th International Forgemasters Meeting, Graz, Austria, 2017.

[7] Vrana J., Goldammer M. "Induction and Conduction Thermography: From the Basics to Application". Thermografie-Anwenderkonferenz, Munich, Germany, 2017, DOI: 10.13140/RG.2.2.13650.86728.

[8] Riegert G., Busse G., "Induktions-Lockin-Thermografie". MP Materialprüfung, vol. 46, pp. 33-35, 2004.

[9] Sakagami T., Ogura K., Kubo S., "Development of Thermographic NDT for the Damage Inspection in Carbon Fiber Reinforced Plastics". The First US Japan Symposium on Advances in NDT, pp. 420-425, 1996. 